\title{
Influence of transverse loading on the stability of a clamped rectangular plate
}

\author{
Mikhail Sukhoterin ${ }^{1, *}$, Sergey Baryshnikov ${ }^{1}$, Tatiana Knysh $^{1}$ and Natalia Pizhurina ${ }^{1}$ \\ ${ }^{1}$ Admiral Makarov State University of Maritime and Inland Shipping, 5/7 Dvinskaya str., 198035, St. \\ Petersburg, Russia
}

\begin{abstract}
Shapes of a square Kirchhoff plate with a clamped edge are obtained and analyzed, before and after losing stability in the case of a compound bending (uniform transverse loading in combination with edge compressive loading), as well as equilibrium forms and critical loadings only with clamping in the plate's surface. Hyperbolic trigonometric series are used for solving. It was established that transverse loading causing small deformations does not affect the plate's stability. The range of the critical state corresponds with an unlimited increase in bends of interior points of a plate. As critical loading, we suggest taking the one at which the bends at the plate's center tend to infinity the most rapidly. As balanced loading, we suggest taking the one at which the plate acquires a new stable equilibrium form. A range of critical and balanced loadings of a square plate with a clamped edge was presented. The corresponding 3D forms of supercritical equilibrium of the given plate were obtained. A comparison with the results of other authors is given.
\end{abstract}

\section{Introduction}

In many constructions, flat rectangular elements - plates (slabs, panels) with the whole edge clamped - are used. These elements can be under transverse loading or compressive loading, applied to the edge in the element's surface. The action of compressive loading can lead to losing stability. Plates are often in the conditions of a compound bending, i.e. joint action of compressive and transverse loadings. Deflections and deflection angles should become zero at the clamped edges, which makes the boundary problem much more complicated than for the case of hinge support of the sides. The loss in stability of separate elements during the first (Eulerian) critical loading is often the main reason for their destruction and the destruction of the whole construction. However, in some cases, with fast passing of the critical loading or with the presence of restrictors (e.g. surfaces), elastic elements can avoid destruction and acquire a new form of stable equilibrium, i.e. can resist supercritical loadings. Thus, it is important, along with determining the first critical loading, to know several following loadings (range) especially for prospective materials with high elastic properties.

It is generally accepted that the loss of stability happens instantaneously when reaching a critical loading and the plate acquires a new equilibrium form also instantaneously, i.e.

\footnotetext{
*Corresponding author: $\underline{\text { mv@sukhoterin.com }}$
} 
critical and balanced loadings coincide, although the loss of stability should come with an unlimited increase in bendings and only then, if no destruction has occurred (in the case of the material having high elastic properties), the plate comes over to a new equilibrium state with the loading beyond critical. Certainly, the increase in bendings should be spread over time and loading but in fact, destruction does not happen instantaneously. There is always accumulation of deflections and then destruction. That is why we should separate the notions of critical and balanced loadings for a plate with a clamped edge. Moreover, we can speak of some critical interval of the compressive loading changing when bendings become greater than the plate's thickness. The loading should be taken as critical, at which the bendings will increase the most rapidly (maximum of potential energy). At the end of this interval, after passing the critical loading and with a decrease in bendings, balanced loading will lead to the new equilibrium state corresponding with the minimum of the plate's potential energy.

Historically, the research on stability of a clamped square plate began with the main problem of stability from the action of compressive loading only. Many papers were focused only on determining the Eulerian loading.

In the paper by Sezawa [1], the solution of the main stability problem was chosen as a sum of two trigonometrical series in two coordinates not resulting in bending at the edge. However, zero turns of clamped edges were found only in the corners of the plate and in centers of its sides. From these conditions, the series coefficients were found. In the paper by Taylor [2], the solution [1] was specified by the collocations method. Upon this, turning angles of the plate's side planes were made equal to zero in a greater number of points than in [1]. Critical forces of a rectangular plate with a clamped edge (the precision of which was not analyzed) were found by Faxen [3] using the strain-energy method [1]. Timoshenko [4] used the strain-energy method to solve this problem as well. Darevskaya [5] applied quartic polynomials and trigonometric series for the solution. The problem was reduced to solving an infinite system relative to unknown coefficients. The first three critical loadings for an equally clamped square plate were obtained. In papers of the last years [6-14], various approximate methods were used to solve the stability problem of a rectangular clamped plate, however, only the first critical loading was looked for.

The goal of this paper is studying the influence of a transverse loading on the stability of a rectangular clamped plate, determining to a high precision the range of critical and balanced compressing forces and the corresponding forms of elastic supercritical equilibrium.

\section{Stability in the case of a compound bending}

Let us assume that the plate is rigid, which means that transverse loading causes small deformations. The differential equation of the bending of rectangular plate $a \times b$, under uniform transverse loading of intensity $q_{0}$ and evenly distributed compressive forces $T_{x}$ and $T_{y}$, applied at the plate's edges, has the form of [15]:

$$
D \nabla^{2} \nabla^{2} W=q_{0}-T_{x} \frac{\partial^{2} W}{\partial X^{2}}-T_{y} \frac{\partial^{2} W}{\partial Y^{2}},
$$

where $W(X, Y)$ - deflection of the median surface, $D=E H^{3} /\left[12\left(1-v^{2}\right)\right]$ - bending rigidity, $H$ - constant thickness of the plate, $E$ - Young's modulus, $v$ - Poisson's ratio, $\nabla^{2}-$ twodimensional Laplace operator.

If we introduce dimensionless coordinates $x=X / b, y=Y / b$, then equation (1) will be written over as 


$$
\nabla^{2} \nabla^{2} w+N_{x} \frac{\partial^{2} w}{\partial x^{2}}+N_{y} \frac{\partial^{2} w}{\partial y^{2}}=1
$$

where $w=W D /\left(q_{0} b^{4}\right)$ - relative deflection, $N_{x}=T_{x} b^{2} / D, \quad N_{y}=T_{y} b^{2} / D$ - relative compressive forces.

Let us put the origin of coordinates at the center of the plate, the measurements of which will now be:'

Let us introduce the boundary conditions of a rigid edge constraint:

$$
\begin{aligned}
& x= \pm \gamma / 2: \quad w=0, \quad \partial w / \partial x=0 \\
& y= \pm 1 / 2: \quad w=0, \quad \partial w / \partial y=0
\end{aligned}
$$

\subsection{Methods and Materials}

Let us expand the single loading in the right part of equation (2) into a double Fourier series:

$$
1=\frac{16}{\gamma} \sum_{k=1,3, \ldots}^{\infty} \sum_{s=1,3, \ldots}^{\infty}(-1)^{\tilde{k}+1}(-1)^{\tilde{s}+1} \frac{\cos \mu_{s} x}{\mu_{s}} \frac{\cos \lambda_{k} y}{\lambda_{k}},
$$

Where $\mu_{s}=s \pi / \gamma, \quad \lambda_{k}=k \pi, \tilde{k}=(k+1) / 2, \quad \tilde{s}=(s+1) / 2$.

The specific solution of equation (2), not giving deflections at the edge, taking into account expanding (5) will have the form of:

$$
w_{0}(x, y)=\frac{16}{\gamma} \sum_{k=1,3, \ldots}^{\infty} \sum_{s=1,3, \ldots}^{\infty} g_{k s} \frac{(-1)^{\tilde{k}}(-1)^{\tilde{s}}}{\lambda_{k} \mu_{s}} \cos \mu_{s} x \cos \lambda_{k} y
$$

where

$$
g_{k s}=1 /\left[\left(\lambda_{k}^{2}+\mu_{s}^{2}\right)^{2}-N_{x} \mu_{s}^{2}-N_{y} \lambda_{k}^{2}\right]
$$

Function (6) breaks the conditions of the clamped edges turns absence (second conditions $(3,4))$, i.e. causes the main "disparities":

$$
\begin{aligned}
& \left.\theta_{x 0}\right|_{x=\frac{\gamma}{2}}=\left.\frac{\partial w_{0}}{\partial x}\right|_{x=\frac{\gamma}{2}}=\sum_{k=1,3, \ldots}^{\infty}(-1)^{\tilde{k}+1} a_{k 0} \cos \lambda_{k} y \\
& \left.\theta_{y 0}\right|_{y=\frac{1}{2}}=\left.\frac{\partial w_{0}}{\partial y}\right|_{y=\frac{1}{2}}=\sum_{s=1,3, \ldots}^{\infty}(-1)^{\tilde{s}+1} b_{s o} \cos \mu_{s} x
\end{aligned}
$$

where

$$
a_{k 0}=-\frac{16}{\gamma \lambda_{k}} \sum_{s=1,3, \ldots}^{\infty} g_{k s}, \quad b_{s 0}=-\frac{16}{\gamma \mu_{s}} \sum_{k=1,3, \ldots}^{\infty} g_{k s}
$$

Let us note that at the opposing edges, formulae (8) and (9) will change sign.

Disparities (8), (9), and the following ones were removed during the infinite iteration process of superposition of two kinds of correcting functions 


$$
\begin{gathered}
w_{1 n}(x, y)=\sum_{k=1,3, \ldots}^{\infty}(-1)^{\tilde{k}+1}\left(\operatorname{ch} \alpha_{k} x-\frac{\operatorname{ch} \tilde{\alpha}_{k}}{\operatorname{ch} \tilde{\beta}_{k}} \operatorname{ch} \beta_{k} x\right) A_{k n} \cos \lambda_{k} y, \\
w_{2 n}(x, y)=\sum_{s=1,3, \ldots}^{\infty}(-1)^{\tilde{s}+1}\left(\operatorname{ch} \xi_{s} y-\frac{\operatorname{ch} \tilde{\xi}_{s}}{\operatorname{ch} \tilde{\eta}_{s}} \operatorname{ch} \eta_{s} y\right) B_{s n} \cos \mu_{s} x
\end{gathered}
$$

by means of coefficients $A_{k n}, B_{s n}$. Here, $n$-iteration index,

$$
\begin{gathered}
\alpha_{k}, \beta_{k}=\sqrt{0,5\left[2 \lambda_{k}^{2}-N_{x} \pm \sqrt{4 \lambda_{k}^{2}\left(N_{y}-N_{x}\right)+N_{x}^{2}}\right]} \\
\xi_{s}, \eta_{s}=\sqrt{0,5\left[2 \mu_{s}^{2}-N_{y} \pm \sqrt{4 \mu_{s}^{2}\left(N_{x}-N_{y}\right)+N_{y}^{2}}\right]} \\
\tilde{\alpha}_{k}=\alpha_{k} \gamma / 2, \quad \tilde{\beta}_{k}=\beta_{k} \gamma / 2, \quad \xi_{s}=\xi_{s} / 2, \quad \tilde{\eta}_{s}=\eta_{s} / 2
\end{gathered}
$$

Functions (11), (12) due to ratios (13), (14), are the solution to the corresponding homogenous equation (2) and also do not cause deflections at the edge.

Let us note that functions of deflections w0, w1n and $\mathrm{w} 2 \mathrm{n}$ are chosen symmetric relative to the origin of coordinates by virtue of the transverse loading symmetry. It is justified when we speak of compressive loadings not exceeding the Eulerian loading. With an increase in supercritical loading, not only symmetric equilibrium forms can be expected to occur, but also antisymmetric and mixed ones. Here, only the symmetric version of the solution will be considered. It can be assumed that after the first loss of stability, transverse loading (causing small deformations of the bending) will not further influence the plate's stability and can be ignored.

Making up for disparity (8) by series (11), we will obtain (the first iteration)

$$
A_{k 1}=-\frac{a_{k 0}}{\tau_{k} \operatorname{ch} \tilde{\alpha}_{k}},\left(\tau_{k}=\alpha_{k} \text { th } \tilde{\alpha}_{k}-\beta_{k} \text { th } \tilde{\beta}_{k}\right)
$$

Function (11) at the edges $y= \pm 1 / 2$ causes angular deformations of clamped sections, i.e. leads to disparities similar to (9)

$$
\left.\theta_{y 1}\right|_{y=\frac{1}{2}}=\left.\frac{\partial w_{11}}{\partial y}\right|_{y=\frac{1}{2}}=-\sum_{k=1,3, \ldots}^{\infty} \lambda_{k}\left(\operatorname{ch} \alpha_{k} x-\frac{\operatorname{ch} \tilde{\alpha}_{k}}{\operatorname{ch} \tilde{\beta}_{k}} \operatorname{ch} \beta_{k} x\right) A_{k 1}
$$

which should be expanded into Fourier series by cosines along with changing the summation signs and transform to the form of:

$$
\left.\theta_{y 1}\right|_{y=\frac{1}{2}}=\sum_{s=1,3, \ldots}^{\infty}(-1)^{s+1} b_{s 1} \cos \mu_{s} x
$$

where

$$
b_{s 1}=-\frac{4 \mu_{s}}{\gamma} \sum_{k=1,3, \ldots}^{\infty} \frac{g_{k s} \lambda_{k} \sqrt{4 \lambda_{k}^{2}\left(N_{y}-N_{x}\right)+N_{x}^{2}}}{\tau_{k}} a_{k 0} \sqrt{2}
$$

Let us add up disparities (9) and (18) now: 


$$
\left.\theta_{y 01}\right|_{y=\frac{1}{2}}=\sum_{s=1,3, \ldots}^{\infty}(-1)^{\tilde{s}+1} b_{s 01} \cos \mu_{s} x,\left(b_{s 01}=b_{s 0}+b_{s 1}\right)
$$

Making up for disparities (20) by series (12), we will obtain

$$
B_{s 1}=-\frac{b_{s 01}}{\omega_{s} \operatorname{ch} \tilde{\xi}_{s}},\left(\omega_{s}=\xi_{s} \text { th } \tilde{\xi}_{s}-\eta_{s} \text { th } \tilde{\eta}_{s}\right)
$$

Function (12) at the edges $x= \pm \gamma / 2$ also causes angular deformations

$$
\left.\theta_{x 1}\right|_{x=\gamma / 2}=\left.\frac{\partial w_{21}}{\partial x}\right|_{x=\gamma / 2}=-\sum_{s=1,3, \ldots}^{\infty} \mu_{s}\left(\operatorname{ch} \xi_{s} y-\frac{\operatorname{ch} \tilde{\xi}_{s}}{\operatorname{ch} \tilde{\eta}_{s}} \operatorname{ch} \eta_{s} y\right) B_{s 1}
$$

which we will similarly expand to the Fourier series by cosines:

$$
\left.\theta_{x 1}\right|_{x=\gamma / 2}=\sum_{k=1,3, \ldots}^{\infty}(-1)^{\tilde{k}+1} a_{k 1} \cos \lambda_{k} y
$$

where

$$
a_{k 1}=-4 \lambda_{k} \sum_{s=1,3, \ldots}^{\infty} \frac{g_{k s} \mu_{s} \sqrt{4 \mu_{s}^{2}\left(N_{x}-N_{y}\right)+N_{y}^{2}}}{\omega_{s}} b_{s 01}
$$

Next, functions $w_{12}(x, y), w_{22}(x, y)$ etc. are used again.

Full removal of disparities is achieved by infinite application of correcting functions during the iteration process which can be easily implemented with a computer. The disparities should decrease in absolute value: $a_{k n}, b_{s n} \rightarrow 0$ when $n \rightarrow \infty$. When reaching the defined calculation accuracy, the iteration process stops.

The desired solution can now be written as:

$$
w(x, y)=w_{0}(x, y)+\sum_{n=1}^{\infty} w_{1 n}(x, y)+w_{2 n}(x, y)
$$

Let us consider the case of equal compressing forces from two directions $N_{x}=N_{y}=N$ . The calculating formulae will take the form of:

$$
\begin{gathered}
g_{k s}=1 /\left[\left(\lambda_{k}^{2}+\mu_{s}^{2}\right)\left(\lambda_{k}^{2}+\mu_{s}^{2}-N\right)\right], \quad \alpha_{k}=\lambda_{k}, \quad \beta_{k}=\sqrt{\lambda_{k}^{2}-N} \\
\xi_{s}=\mu_{s}, \quad \eta_{s}=\sqrt{\mu_{s}^{2}-N}, \quad a_{k 0}=-\frac{16}{\gamma \lambda_{k}} \sum_{s=1,3, \ldots}^{\infty} g_{k s}, \quad b_{s 0}=-\frac{16}{\gamma \mu_{s}} \sum_{k=1,3, \ldots}^{\infty} g_{k s} \\
a_{k n}=-4 \lambda_{k} N \sum_{s=1,3, \ldots}^{\infty} \frac{g_{k s} \mu_{s} b_{s n}}{\omega_{s}}, \quad b_{s n}=-\frac{4 N}{\gamma} \mu_{s} \sum_{k=1,3, \ldots}^{\infty} \frac{g_{k s} \lambda_{k} a_{k(n-1)}}{\tau_{k}}
\end{gathered}
$$

If we substitute the last expression (changing index $k$ to $\mathrm{m}$ ) into the previous one, we will obtain

$$
a_{k n}=\frac{16 \lambda_{k} N^{2}}{\gamma} \sum_{s=1,3, \ldots}^{\infty} \frac{g_{k s} \mu_{s}^{2}}{\omega_{s}} \sum_{m=1,3, \ldots}^{\infty} \frac{g_{m s} \lambda_{m} a_{m(n-1)}}{\tau_{m}}
$$


Thus, coefficients $a_{k n}$ of this iteration are linearly dependent on the collective of similar coefficients $a_{k(\mathrm{n}-1)}$ of the previous iteration, i.e. expression (27) is an infinite homogenous system of linear algebraic equations. If it appears to be regular, the iteration process will be concurrent, the disparities will tend to zero.

Research revealed that starting from the second iteration, coefficients $a_{k n}$ will have order $a_{k n}=\delta_{k n} / \lambda_{k}^{2}$, where $\delta_{k n}$ - some infinitesimal with $k \rightarrow \infty$. Then, system (27) can be written over as

$$
\delta_{k n}=\frac{16 \lambda_{k}^{3} N^{2}}{\gamma} \sum_{s=1,3, \ldots}^{\infty} \frac{g_{k s} \mu_{s}^{2}}{\omega_{s}} \sum_{m=1,3, \ldots}^{\infty} \frac{g_{m s} \delta_{m(n-1)}}{\lambda_{m} \tau_{m}}
$$

For regularity of system (28), $\left|r_{k}\right|<1$ should be fulfilled, where

$$
r_{k}=\frac{16 \lambda_{k}^{3} N^{2}}{\gamma} \sum_{s=1,3, \ldots}^{\infty} \frac{g_{k s} \mu_{s}^{2}}{\omega_{s}} \sum_{m=1,3, \ldots}^{\infty} \frac{g_{m s}}{\lambda_{m} \tau_{m}}
$$

- the regularity factor which contains compressive force $N$ as a parameter.

\subsection{Results}

The regularity factor was calculated in the system of analytical calculations Maple for a large size of changing of parameter $N$.

A square plate was researched in detail. The first three convergence intervals for parameter $N:\left(0,2 \pi^{2}\right),\left(53,10 \pi^{2}\right),(110.2,146)$ and the first two divergence intervals $\left(2 \pi^{2}\right.$, $53),\left(10 \pi^{2}, 110.2\right)$ were obtained. Let us note that there should be an infinite aggregate of such intervals.

The convergence intervals correspond with the plate's stable state and the divergence intervals - with the plate's unstable state.

To calculate bendings, a program in the Maple system was created. The series held up to 499 terms, the number of iterations was up to 30 . With small compressive loading $N=0.01$, the maximum deflection (at the center of the square plate) amounted to 0.0012655 . For reference, the maximum deflection during the action of only a transverse loading in paper [15] amounted to 0.00126 . The deflection line in section $y=0$ is given in fig. 1 .

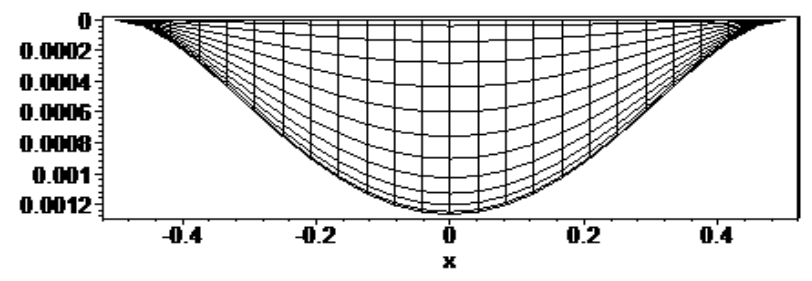

Fig. 1. Deflections in section $\mathrm{y}=0$ of a square plate with $\mathrm{N}=0.01$.

With an increase in compressive forces, deflections also increased, for example, with $N$ $=9$, the maximum deflection amounted to 0.0015332 .

On the interval $\left(53,10 \pi^{2}\right)$, the shape of the plate's bent surface (bulge) already had an opposite direction, i.e. earlier, on the divergence interval $\left(2 \pi^{2}, 53\right)$ there was a loss of stability with a "snap" of the shape in the opposite direction. Calculations with $N=60$ resulted in the maximum deflection of 0.00899 . With $N=90$, the occurrence of four symmetric bulges at the corners of the plate in the opposite direction became noticeable. In the third stability zone $(110.2,146)$, the forming of the surface with five bulges continued. Their direction was the 
same as near the right boundary of interval $\left(53,10 \pi^{2}\right)$. With $N=130$, the maximum deflection at the center amounted to 0.00117 and the maximum deflection of the four opposite bulges amounted to $\approx 0.00025$. The surface shapes were also obtained with $N=175$ and $N=180$, which had five commensurable bulges but of the direction opposite to interval $(110.2,146)$. And this examining process can be continued further.

For a square plate, the ratio of the first formula in (26) for $g_{k s}$ turns to zero with the least value of $N=2 \pi^{2} \approx 19.739$, the next $10 \pi^{2} \approx 98.696$ etc. Here, it should be taken into account that the initial component was the solution of (6) for a simply supported plate. Therefore, these values should be considered special values - the start of the unstable state zone of the plate. Let us note that expressions $\tau_{k}$ and $\omega_{s}$, occurring in ratios of formulae (26), do not turn to zero (except for the case of $N=0$ ). They are always positive and present.

The unstable state zones (divergence intervals of the process) are quite extended. The loss of stability can happen with any value of compressive forces from this interval after their long-term influence. In practice, the loss of stability with forces close to the interval boundaries is unlikely at least because of internal friction in the plate's material.

As the first critical loading for a clamped square plate we suggest accepting the value of $N_{c 1}=37.8$ from the first divergence interval, at which the deflection at the center of the plate was going to infinity the most rapidly with the same number of iterations and where was a change of the deflection sign (snap).

The value of $N_{b 1} \approx 53$ is the start of the new convergence interval (of stable state) and can be called an equilibrium value. With this value, a new stable equilibrium form with the opposite bulging is observed. Similarly were found the second critical loading $N_{c 2}=104$ and the second equilibrium value $N_{b 2} \approx 110.2$. A strict research on the intervals of unstable state should be done within the context of the nonlinear theory.

In fig. 2-4, stable shapes of the bent surface of a square plate with a compound bending for various values of compressive loading are given.

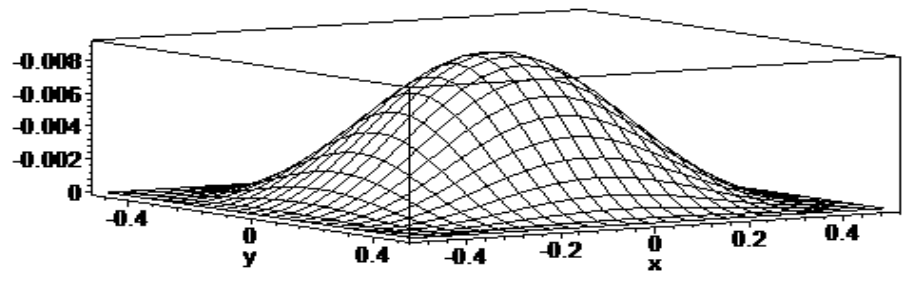

Fig. 2. Shape of the square plate with a compound bending for $N=60$.

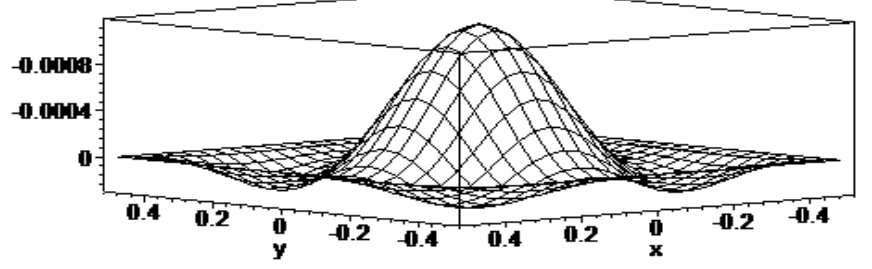

Fig. 3. Shape of the square plate with a compound bending for $\mathrm{N}=130$. 


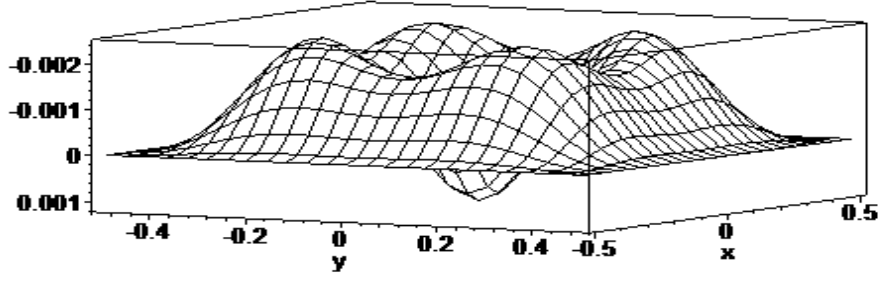

Fig. 4. Shape of the square plate with a compound bending for $N=175$.

\section{The main stability problem}

In this case, only compressing forces are acting, transverse loading is absent, and the equation of bending (2) will be homogeneous:

$$
\nabla^{2} \nabla^{2} w+N_{x} \frac{\partial^{2} w}{\partial x^{2}}+N_{y} \frac{\partial^{2} w}{\partial y^{2}}=0
$$

The solution can be symmetric, antisymmetric, and mixed, leading to the corresponding equilibrium forms and critical loadings.

\subsection{Methods and Materials}

The symmetric solution (S) we write, like in the case of a compound bending, as

$$
\begin{array}{r}
w_{1 \mathrm{~S}}(x, y)=\sum_{k=1,3, \ldots}^{\infty}(-1)^{\tilde{k}}\left(\operatorname{ch} \alpha_{k} x-\frac{\operatorname{ch} \tilde{\alpha}_{k}}{\operatorname{ch} \tilde{\beta}_{k}} \operatorname{ch} \beta_{k} x\right) A_{k} \cos \lambda_{k} y \quad\left(\lambda_{k}=k \pi\right) \\
w_{2 \mathrm{~S}}(x, y)=\sum_{s=1,3, \ldots}^{\infty}(-1)^{\tilde{s}}\left(\operatorname{ch} \xi_{s} y-\frac{\operatorname{ch} \tilde{\xi}_{s}}{\operatorname{ch} \tilde{\eta}_{s}} \operatorname{ch} \eta_{s} y\right) B_{s} \cos \mu_{s} x, \quad\left(\mu_{s}=s \pi / \gamma\right)
\end{array}
$$

antisymmetric (A)

$$
\begin{aligned}
& w_{1 \mathrm{~A}}(x, y)=\sum_{k=1}^{\infty}(-1)^{k}\left(\operatorname{sh} \alpha_{k} x-\frac{\operatorname{sh} \tilde{\alpha}_{k}}{\operatorname{sh} \tilde{\beta}_{k}} \operatorname{sh} \beta_{k} x\right) A_{k} \sin \lambda_{k} y \quad, \quad\left(\lambda_{k}=2 k \pi\right) \\
& w_{2 \mathrm{~A}}(x, y)=\sum_{s=1}^{\infty}(-1)^{s}\left(\operatorname{sh} \xi_{s} y-\frac{\operatorname{sh} \tilde{\xi}_{s}}{\operatorname{sh} \tilde{\eta}_{s}} \operatorname{sh} \eta_{s} y\right) B_{s} \sin \mu_{s} x,\left(\mu_{s}=2 s \pi / \gamma\right)
\end{aligned}
$$

antisymmetric-symmetric (AS)

$$
\begin{gathered}
w_{1 \mathrm{AS}}(x, y)=\sum_{k=1,3, \ldots}^{\infty}(-1)^{\tilde{k}}\left(\operatorname{sh} \alpha_{k} x-\frac{\operatorname{sh} \tilde{\alpha}_{k}}{\operatorname{sh} \tilde{\beta}_{k}} \operatorname{sh} \beta_{k} x\right) A_{k} \cos \lambda_{k} y, \quad\left(\lambda_{k}=k \pi\right) \\
w_{2 \mathrm{AS}}(x, y)=\sum_{s=1}^{\infty}(-1)^{s}\left(\operatorname{ch} \xi_{s} y-\frac{\operatorname{ch} \tilde{\xi}_{s}}{\operatorname{ch} \tilde{\eta}_{s}} \operatorname{ch} \eta_{s} y\right) B_{s} \sin \mu_{s} x, \quad\left(\mu_{s}=2 s \pi / \gamma\right)
\end{gathered}
$$

symmetric-antisymmetric (AS) 


$$
\begin{array}{r}
w_{\mathrm{ISA}}(x, y)=\sum_{k=1}^{\infty}(-1)^{k}\left(\operatorname{ch} \alpha_{k} x-\frac{\operatorname{ch} \tilde{\alpha}_{k}}{\operatorname{ch} \tilde{\beta}_{k}} \operatorname{ch} \beta_{k} x\right) A_{k} \sin \lambda_{k} y,\left(\lambda_{k}=2 k \pi\right) \\
w_{2 \mathrm{SA}}(x, y)=\sum_{s=1,3, \ldots}^{\infty}(-1)^{\tilde{s}}\left(\operatorname{sh} \xi_{s} y-\frac{\operatorname{sh} \tilde{\xi}_{s}}{\operatorname{sh} \tilde{\eta}_{s}} \operatorname{sh} \eta_{s} y\right) B_{s} \cos \mu_{s} x,\left(\mu_{s}=s \pi / \gamma\right)
\end{array}
$$

Functions (31) - (38) comply with equation (30) due to coefficients $\alpha_{k}, \beta_{k}, \xi_{s}, \eta_{s}$, which have the earlier form of (13), (14), and also fulfil the condition of the absence of bendings at the edge.

Let us ask for fulfilling the conditions of the absence of turning angles of the plate's edge. It yields for all four cases an infinite system for unknown coefficients Ak and Bs, which is reduced to a systems with one sequence of coefficients Ak. Let us introduce this system for equal all-around compressing forces $N_{x}=N_{y}=N_{\text {: }}$ :

$$
\tilde{A}_{k}=\frac{16 N^{2}}{\gamma} \frac{\lambda_{k}^{2}}{\tau_{k}} \sum_{s=1}^{\infty} \frac{\mu_{s}^{2}}{\omega_{s}\left(\mu_{s}^{2}+\lambda_{k}^{2}\right)\left(\mu_{s}^{2}+\lambda_{k}^{2}-N\right)} \sum_{m=1}^{\infty} \frac{\tilde{A}_{m}}{\left(\lambda_{m}^{2}+\mu_{s}^{2}\right)\left(\lambda_{m}^{2}+\mu_{s}^{2}-N\right)}
$$

here, for the symmetric solution:

$$
\begin{gathered}
A_{k}=\tilde{A}_{k} /\left(\lambda_{k} \operatorname{ch} \tilde{\alpha}_{k}\right), \quad k, m, s=1,3, \ldots, \\
\tau_{k}=\alpha_{k} \text { th } \tilde{\alpha}_{k}-\beta_{k} \text { th } \tilde{\beta}_{k}, \omega_{s}=\xi_{s} \text { th } \tilde{\xi}-\eta_{s} \text { th } \tilde{\eta}_{s}
\end{gathered}
$$

for the antisymmetric solution:

$$
\begin{gathered}
A_{k}=\tilde{A}_{k} /\left(\lambda_{k} \operatorname{sh} \tilde{\alpha}_{k}\right), \quad k, m, s=1,2, \ldots, \\
\tau_{k}=\alpha_{k} \operatorname{cth} \tilde{\alpha}_{k}-\beta_{k} \operatorname{cth} \tilde{\beta}_{k}, \omega_{s}=\xi_{s} \operatorname{cth} \tilde{\xi}-\eta_{s} \operatorname{cth} \tilde{\eta}_{s}
\end{gathered}
$$

for the antisymmetric-symmetric solution:

$$
\begin{gathered}
A_{k}=\tilde{A}_{k} /\left(\lambda_{k} \operatorname{sh} \tilde{\alpha}_{k}\right), \quad k, m=1,3, \ldots, s=1,2, \ldots \\
\tau_{k}=\alpha_{k} \operatorname{th} \tilde{\alpha}_{k}-\beta_{k} \operatorname{th} \tilde{\beta}_{k}, \omega_{s}=\xi_{s} \operatorname{cth} \tilde{\xi}-\eta_{s} \operatorname{cth} \tilde{\eta}_{s}
\end{gathered}
$$

for the symmetric-antisymmetric solution:

$$
\begin{gathered}
A_{k}=\tilde{A}_{k} /\left(\lambda_{k} \operatorname{ch} \tilde{\alpha}_{k}\right), \quad k, m=1,2, \ldots, s=1,3, \ldots, \\
\tau_{k}=\alpha_{k} \operatorname{cth} \tilde{\alpha}_{k}-\beta_{k} \operatorname{cth} \tilde{\beta}_{k}, \omega_{s}=\xi_{s} \operatorname{th} \tilde{\xi}-\eta_{s} \operatorname{th} \tilde{\eta}_{s}
\end{gathered}
$$

System (39) contains compressive loading $\mathrm{N}$ as a parameter and represents an infinite homogenous system of linear algebraic equations with respect to coefficients $\tilde{A}_{k}$, written in the canonical form. This form allows organizing the iteration process of its solution, considering coefficients $\tilde{A}_{m}$ as coefficients of the previous iteration and coefficients $\tilde{A}_{k}$ - as of the following one. 


\subsection{Results}

A program for solving reduced system (39) was created in the Maple system. All initial values of coefficients $\tilde{A}_{m}$ were taken equal to unity. The size of the reduced system (the number of series terms) was equal to 59. It provided for high precision of calculations taking into account convergence of series. The number of iterations - up to 50. Loading $\mathrm{N}$ varied over wide range with changing increments. Its values, with which nontrivial final solutions of system (39) were obtained, were taken as equilibrium values. The corresponding equilibrium forms were obtained for a range of these loadings. The research for each of the four types of solution allowed finding intervals of stable state, when the iteration process led to trivial solutions, as well as intervals of unstable state, when coefficients unlimitedly increased with an increase in the number of iterations (deflections increased as well). The rate of coefficients' increase depended on loading with the same number of iterations. The values taken as critical were those, with which the coefficients were going to infinity the most rapidly.

A square plate was researched in detail. In the case of the symmetric solution, the results were compared with the results in the case of a compound bending. In particular, the first critical values 37.8 were completely equal. The second values were quite close: 104 and 104.289. The first equilibrium forces: 53 and 52.345 (one bulge). We did not find a pair for the second equilibrium force 110.2 in the case of a compound bending. The second equilibrium value in the main stability problem appeared to be equal to 167.03 . The surface shape had one central bulge and four bulges close to the plate's corners.

The calculations allowed obtaining the initial range of critical loadings for a square plate: 37.8 (S - solution); 58.47 (AS); 85.081 (AS); 104.289 (S); 105.119 (A); 147.291 (AS); 156.25 (S); 211.795 (A). The initial range of equilibrium forces was also obtained: 52.345 (S - form); 92.125 (AS); 128.121 (A); 167.03 (S) and the corresponding three-dimensional supercritical equilibrium forms.

In fig. 5-8, the first four equilibrium forms of an evenly compressed square plate are given.

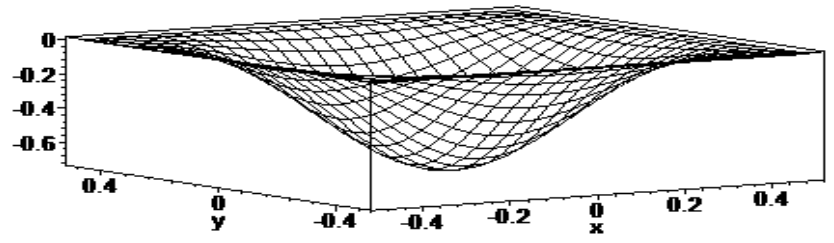

Fig. 5. Symmetric equilibrium form of the square plate with $\mathrm{N}=52.345$.

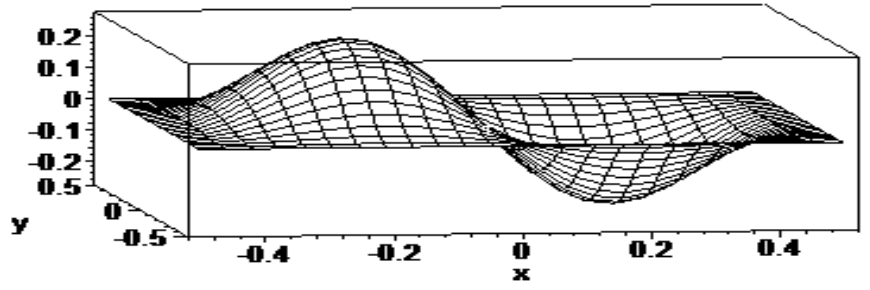

Fig. 6. Antisymmetric-symmetric equilibrium form of the square plate with $\mathrm{N}=92.125$. 


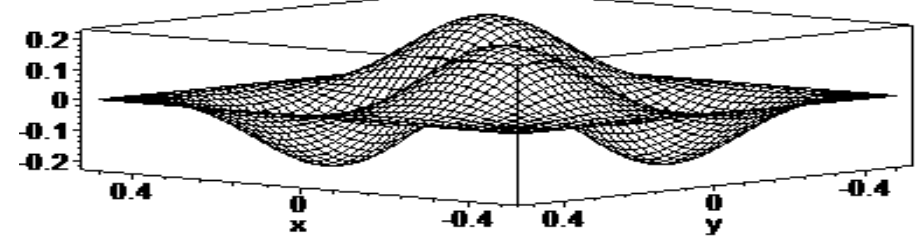

Fig. 7. Antisymmetric equilibrium form of the square plate with $\mathrm{N}=128.21$.

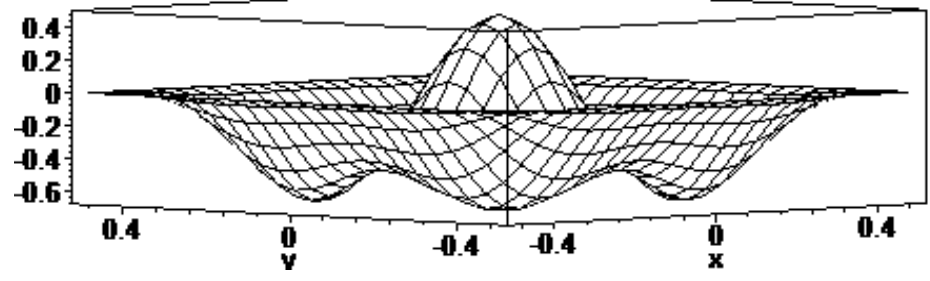

Fig. 8. Symmetric equilibrium form of the square plate with $N=167.03$.

\section{Discussion}

In the paper by Sezawa [1], for a square plate with equal compressive forces on all edges, the first critical force $N_{\ni} \approx 5.61 \pi^{2} \approx 55.37$ (in our interpretation - equilibrium force) was obtained, which is significantly greater than 52.345 obtained in this paper.

In the paper by Taylor [2], the solution [1] was made more precise by the collocation method. The critical loading value $\mathrm{N} э \approx 5.3 \pi 2 \approx 52.31$ was obtained, which appeared to be less than in [1]. Here, a tendency for reducing the critical value in the case of an increase in the number of collocation points is observed. Value 52.31, obtained in [2], is almost the same as our first equilibrium force 52.345 .

In the paper by Timoshenko [4], the equivalent value was 52.638 . He used the strainenergy method, which usually makes the required values conservative.

Darevskaya [5] obtained the first three critical loadings for an evenly compressed square plate (with our designations): 52.388 (S), 97.906 (AS), 148.439 (A). The second and the third critical loadings are significantly different from those obtained in this paper.

Let us note that except for paper [5], there are no other papers in which the second and the following critical loadings were calculated.

In this paper, critical and balanced loadings are calculated with high precision since the main differential equation and the boundary conditions were fulfilled quite precisely. The suggested methods allow obtaining a wide range of the above-noted loadings.

\section{Conclusion}

1. A range of critical and balanced loadings for a square Kirchhoff plate with a clamped edge was obtained in this paper to a high precision.

2. It is suggested to distinguish critical and balanced loadings. Critical loadings are characterized by an unlimited increase in deflections of interior points of a plate. Balanced loadings correspond with a plate reaching equilibrium after the loss of stability.

3. It is discovered that uniform transverse loading, causing small deformations of a plate, does not affect its stability. Transverse loading can be ignored during stability calculations. 
4. The obtained results can be used for practical calculations of durability and stability of rectangular clamped plates with high elastic properties as elements of various constructions as well as for analyzing reasons of their destruction.

The authors express their gratitude to the government for the financial support of this research. The authors would like to gratefully acknowledge the unknown reviewers for their review and helpful comments.

\section{References}

1. K. Sezawa, Z. Angew, Math. Mech. 12, 227-229 (1932) DOI:10.1002/zamm.19320120404

2. G.I. Taylor, Z. Angew, Math. Mech. 13, 147-152 (1933) DOI:10.1002/zamm.19330130222

3. O.H. Faxén, Z. Angew, Math. Mech. 15, 268-277 (1935) DOI: 10.1002/zamm.19350150505

4. S.P. Timoshenko, J.M. Gere, Theory of elastic stability (McGraw-Hill, New York, 1961)

5. E.V. Darevskaya, Struct. Mech. Analys. Construct. 3, 31-35 (1982)

6. H. Nagino, T. Mikami, T. Mizusawa, J. Sound Vibr. 37, 329-353 (2008)

7. Y. Qu, G. Yuan, S. Wu, G. Meng, Europ. J. Mech. A/Solids 42, 376-394 (2013)

8. C.S. Huang, O.G. McGee, K.P. Wang, Int. J. Mech. Sci. 70, 1-25 (2013)

9. O.M. Ibearugbulem, J.C. Ezeh, Academ. Research Intern. 4, 346 - 351 (2013)

10. J.C. Ezeh, O.M Ibearugbulem, H.E. Opara, O.A. Oguaghamba, Intern. J. Research Engin. Technol. 3, 674-679 (2014)

11. S.O. Papkov, J.R. Banerjee, J. Sound Vibr. 339, 342-358 (2015)

12. V.A. Manukhin, V.A. Korshunov, N.N. Viryacheva, The Works of Krylov state research center 373.2(5), 151-160 (2015)

13. S. Papkov, O. Dovgalenko, M. Chevin, J. Innovat. Scienc.(in Russia) 66, 22-26 (2017)

14. S.O. Papkov, Mech. Solids 52, 686-699 (2017) DOI 10.3103/S0025654417060085

15. S. Timoshenko, S. Woinowsky-Krieger, Theory of Plates and Shells (McGraw-Hill Book Company, New York, Toronto, London, 1959) 